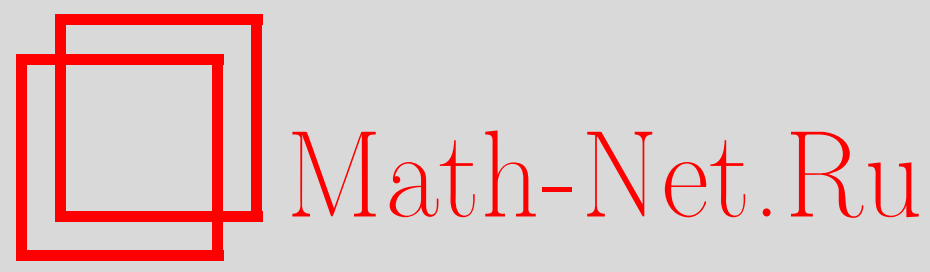

А. В. Покровский, Устранимые особенности слабых решений линейных дифференциальных уравнений с частными производными, Матем. заметки, 2005, том 77, выпуск 4, 584-591

DOI: https://doi.org/10.4213/mzm2519

Использование Общероссийского математического портала Math-Net.Ru подразумевает, что вы прочитали и согласны с пользовательским соглашением http://www . mathnet.ru/rus/agreement

Параметры загрузки:

IP : 54.162 .85 .209

26 апреля 2023 г., $16: 44: 29$

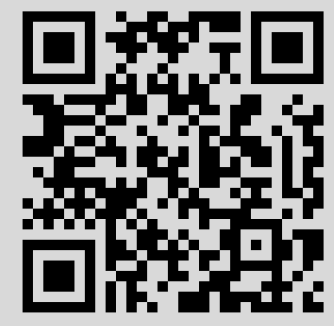




\section{УСТРАНИМЫЕ ОСОБЕННОСТИ СЛАБЫХ РЕШЕНИЙ ЛИНЕЙНЫХ ДИФФЕРЕНЦИАЛЬНЫХ УРАВНЕНИЙ С ЧАСТНЫМИ ПРОИЗВОДНЫМИ}

\section{А. В. Покровский}

Пусть $P(x, D)$ - линейный дифференциальнй оператор порядка $m>0$, коэффициенты которого $m$ раз непрерывно дифференцируемы в области $G \subset \mathbb{R}^{n}(n \geqslant 1)$, и пусть $1<p<\infty, s>0, q=p /(p-1)$. Показано, что если $n, m, p$ и $s$ удовлетворяют двойному неравенству $0 \leqslant n-q(m-s)<n$, то всякое замкнутое в $G$ множество конечной хаусдорфовой меры порядка $n-q(m-s)$ устранимо для слабых решений уравнения $P(x, D) u=0$ в классе Шарпли-ДеВора $C_{p}^{s}(G)_{\text {loc. }}$ Это усиливает известный результат Р. Харви и Дж. Полкинга об устранимых особенностях слабых решений уравнения $P(x, D) u=0$ в классах Соболева и распространяет его на нецелые показатели гладкости.

Библиография: 11 названий.

Введение. Пусть $G$ - область в $\mathbb{R}^{n}, n \geqslant 1$,

$$
P(x, D)=\sum_{|k| \leqslant m} a_{k}(x) D^{k}
$$

- линейньй дифференциальньй оператор порядка $m>0$, коэффициенты $a_{k}$ которого $m$ раз непрерьвно дифференцируемые функции в области $G$ (здесь и далее рассматриваются функции, принимающие, вообще говоря, комплексные значения): $a_{k} \in C^{m}(G)$, $|k| \leqslant m$. Здесь $k=\left(k_{1}, \ldots, k_{n}\right) \in \mathbb{N}_{0}^{n}, \mathbb{N}_{0}:=\{0,1, \ldots\}$,

$$
|k|=k_{1}+\cdots+k_{n}, \quad D^{k}=\frac{\partial^{|k|}}{\partial^{k_{1}} x_{1} \cdots \partial^{k_{n}} x_{n}} .
$$

Пусть $\Omega$ - непустое открытое подмножество области $G$. Как обычно, $C_{0}^{\infty}(\Omega)$ - множество всех функций, бесконечное число раз дифференцируемых в $\mathbb{R}^{n}$, носитель которых компакт, лежаший в $\Omega$. Функция $f$, локально интегрируемая по Лебегу на множестве $\Omega$, назьвается слабым решением уравнения

$$
P(x, D) u=0
$$

Работа вьполнена при частичной поддержке Государственной программы Украины, грант № 0102 U000917. 
на этом множестве, если для любой функции $\phi \in C_{0}^{\infty}(\Omega)$ выполяется равенство

$$
(P(x, D) f, \phi):=\sum_{|k| \leqslant m}(-1)^{|k|} \int_{\Omega} f(x) D^{k}\left(a_{k} \phi\right)(x) d x=0 .
$$

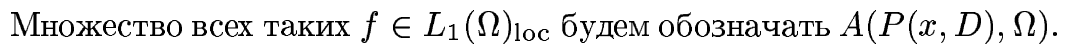

Пусть $1<p<\infty, s \in \mathbb{N}_{0}$. Как обычно, $W_{p}^{s}(G)_{\text {lос }}$ - множество всех функций, имеющих обобщенные производные по Соболеву всех порядков $\leqslant s$, локально интегрируемые в степени $p, W_{p}^{0}(G)_{\text {lос }}:=L_{p}(G)_{\text {lос }}$. Пусть $q=p(p-1)^{-1}$, а $s$ таково, что вьполнено двойное неравенство $0 \leqslant n-q(m-s)<n$. При сделанных предположениях в работе [1] установлен следующий результат о стирании особенностей слабых решений уравнения $P(x, D) u=0$ в классе $W_{p}^{s}(G)_{\text {lос }}$.

Tеорема 1 (см. [1], а также [2, с. 23]). Пусть $E$ - замкнутое мнохсество в $G$, имеющее конечную хаусдорфову меру порядка $n-q(m-s): \operatorname{mes}^{n-q(m-s)} E<\infty$. Тогда справедливо включение

$$
A(P(x, D), G \backslash E) \cap W_{p}^{s}(G)_{\mathrm{loc}} \subset A(P(x, D), G) .
$$

При этом в [1] на примерах показано, что в этой теореме показатель $n-q(m-s)$ увеличить нельзя.

В настоящей заметке доказьвается, что утверждение теоремы 1 остается справедли-

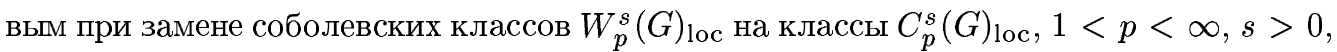
введенные Р. Шарпли и Р. ДеВором в работе [3] и независимо Б. Боярским в работе [4]. Поскольку в случае натурального $s$ всегда справедливо включение

$$
W_{p}^{s}(G)_{\mathrm{loc}} \subset C_{p}^{s}(G)_{\mathrm{loc}}
$$

это усиливает приведенную выше теорему 1 и распространяет ее на нецелые показатели гладкости $s$.

Основной результат работы впервые доложен на Международной конференции “Функциональные пространства. Дифференциальные операторы. Проблемы математического образования", см. [5].

1. Определения, формулировка основного результата, вспомагательные предложения. Всюду ниже выражения вида $C(\alpha, \beta, \ldots)$ обозначают действительную неотрищательную величину, зависящюю только от $\alpha, \beta$ и т.д.

Если $x \in \mathbb{R}^{n}$ и $r>0$, то $|x|$ - евклидова норма вектора $x$,

$$
B(x, r):=\left\{y \in \mathbb{R}^{n}:|x-y|<r\right\} .
$$

Пусть $1<p<\infty, s>0, f$ - функция, локально суммируемая в области $G$ : $f \in$ $L_{1}(G)_{\text {lос. }}$ Возьмем замкнутьй куб $Q(x, r)$ с центром в точке $x$ и длиной ребра $r>0$, лежащий в области $G$, стороны которого параллельны координатным осям, и обозначим

$$
E_{[s]}(f, x, r):=\inf \left\{r^{-n} \int_{Q(x, r)}|f(y)-g(y)| d y: g \in \mathscr{P}_{[s]}\right\}
$$


где $[s]$ - целая часть $s, \mathscr{P}_{[s]}$ - множество всех алгебраических полиномов степени не выше $[s]$ (по совокупности переменных). Определим в области $G$ максимальную функцию $M_{s} f$ равенством

$$
M_{s} f(x):=\sup _{Q(x, r) \subset G} r^{-s} E_{|s|}(f, x, r)
$$

По определению функция $f$ принадлежит классу $C_{p}^{s}(G)_{\text {loc }}$, если функция $M_{s} f$ локально суммируема в степени $p$ в области $G: M_{s} f \in L_{p}(G)_{\text {loc }}$.

Сразу же отметим, что последнее условие обеспечивает принадлежность $f$ к $L_{p}(G)_{\text {loc }}$, что согласуется с определением из работы [3]. Для доказательства этого зафиксируем функцию $\psi \in C_{0}^{\infty}\left(\mathbb{R}^{n}\right)$ такую, что носитель $\psi$ содержится в шаре $|x| \leqslant 1$,

$$
\int_{|x| \leqslant 1} \psi(x) d x=1 \quad \text { и } \quad \int_{|x| \leqslant 1} g(x) \psi(x) d x=g(0)
$$

для любого полинома $g \in \mathscr{P}_{[s]}$ (существование такой функции вытекает, например, из результатов работы [6], см. также [7]). Пусть $G_{1}$ - подобласть области $G$ такая, что $G_{1} \Subset G$. Рассмотрим соболевскую регуляризацию

$$
\begin{gathered}
f_{r}(x):=\int_{|y| \leqslant 1} f(x-r y) \psi(y) d y, \quad x \in G_{1}, \quad 0<r<(\sqrt{n})^{-1} \operatorname{dist}\left(\partial G_{1}, \partial G\right), \\
\operatorname{dist}\left(\partial G_{1}, \partial G\right):=\inf \left\{|x-y|: x \in \partial G_{1}, y \in \partial G\right\}>0 .
\end{gathered}
$$

Тогда

$$
\begin{aligned}
\left|f_{r / 2}(x)-f_{r / 4}(x)\right|= & \inf _{g \in \mathscr{P}_{[s]} \mid} \mid \int_{|y| \leqslant 1}\left(f\left(x-2^{-1} r y\right)-g\left(x-2^{-1} r y\right)\right) \psi(y) d y \\
& \quad-\int_{|y| \leqslant 1}\left(f\left(x-4^{-1} r y\right)-g\left(x-4^{-1} r y\right)\right) \psi(y) d y \mid \\
\leqslant & C(n, \psi) E_{[s]}(f, x, r),
\end{aligned}
$$

откуда

$$
\begin{aligned}
\left(\int_{G_{1}}\left|f_{r / 2}(x)-f_{r / 4}(x)\right|^{p} d x\right)^{1 / p} & \leqslant C(n, \psi)\left(\int_{G_{1}}\left(E_{[s]}(f, x, r)\right)^{p} d x\right)^{1 / p} \\
& \leqslant C(n, \psi) r^{s}\left(\int_{G_{1}}\left(r^{-s} E_{[s]}(f, x, r)\right)^{p} d x\right)^{1 / p} \\
& \leqslant C(n, \psi) r^{s}\left(\int_{G_{1}}\left(M_{s} f(x)\right)^{p} d x\right)^{1 / p} \\
& =C\left(n, \psi, f, s, G_{1}\right) r^{s} .
\end{aligned}
$$

Положим $r=2^{-l}$, где $l$ пробегает множество натуральных чисел

$$
\geqslant\left[\log _{2}\left(\left(\operatorname{dist}\left(\partial G_{1}, \partial G\right)\right)^{-1} \sqrt{n}\right)\right]+1 .
$$

Тогда из (3) вытекает, что последовательность $f_{2^{-l}}$ фундаментальна в $L_{p}\left(G_{1}\right)$ и, следовательно, сходится по норме этого пространства при $l \rightarrow \infty$ к некоторой функции 
из $L_{p}\left(G_{1}\right)$, которая, как следует из свойств соболевских регуляризаций (см., например, $[8$, с. 65$])$, почти всюду в $G_{1}$ совпадает с функцией $f$. Поскольку в наших рассуждениях область $G_{1}$ может быть выбрана произвольным образом, это означает, что $f \in L_{p}(G)_{\text {loc }}$.

В случае натурального $s$ можно определить в области $G$ еще одну максимальную функцию:

$$
M_{s}^{*} f(x):=\sup _{Q(x, r) \subset G} r^{-s} E_{s}^{*}(f, x, r)
$$

где $E_{s}^{*}(f, x, r)$ обозначает осредненную по мере Лебега величину наименьшего уклонения в среднем функции $f$ на кубе $Q(x, r)$ от пространства алгебраических полино-

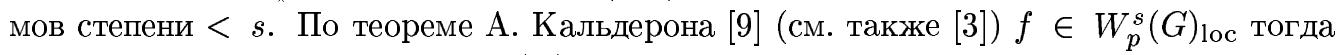
и только тогда, когда $M_{s}^{*} f \in L_{p}(G)_{\text {loc }}$, откуда вытекает упомянутое вьше включение $W_{p}^{s}(G)_{\text {loc }} \subset C_{p}^{s}(G)_{\text {loc }}$.

Пусть $\alpha>0, E$ - множество в $\mathbb{R}^{n}$. Напомним, что (внешней) мерой Хаусдорфа мнохсества $E$ порядка $\alpha$ (обозначение $\operatorname{mes}^{\alpha} E$ ) назьвается конечньй или равный $+\infty$ предел при $\varepsilon \rightarrow 0$ величины $\inf \left(\sum_{i} r_{i}^{\alpha}\right)$, где инфимум берется по всем не более, чем счетным системам открытых шаров $\left\{B\left(x_{i}, r_{i}\right)\right\}_{i}$ с $r_{i} \leqslant \varepsilon$, образующих покрытие множества $E$. Отметим, что величина $\operatorname{mes}^{n} E$ с точностью до постоянного множителя, зависящего только от размерности $n$, совпадает с внешней мерой Лебега множества $E$.

Сформулируем теперь основной результат работы.

Теорема 2. Пусть $1<p<\infty$, as $>0$ таково, что выполнено двойное неравенство $0 \leqslant n-q(m-s)<n$, и пусть $E-$ замкнутое множество в $G$, имеющее конечную хаусдорфову меру порядка $n-q(m-s): \operatorname{mes}^{n-q(m-s)} E<\infty$. Тогда справедливо включение

$$
A(P(x, D), G \backslash E) \cap C_{p}^{s}(G)_{\text {loc }} \subset A(P(x, D), G)
$$

Другими словами, заключение теоремы 2 можно сформулировать так: всякое замкнутое в $G$ множество конечной хаусдорфовой меры порядка $n-q(m-s)$ устранимо для слабых решений уравнения $P(x, D) u=0$ в классе $C_{p}^{s}(G)_{\text {loc }}$. Из результатов работы $[10]$ (теорема 4) вытекает, что если $P(x, D)=P(D)=\sum_{|k|=m} a_{k} D^{k}$ - однородньй эллиптический оператор с постоянньми коэффициентами, то любое замкнутое множество $E$ $(E \neq G)$ хаусдорфовой размерности $>n-q(m-s)$ неустранимо в классе $C_{p}^{s}(G)_{\text {loc }}$, т.е. включение $A(P(D), G \backslash E) \cap C_{p}^{s}(G)_{\text {lос }} \subset A(P(D), G)$ не имеет места. В этом смысле результат теоремы 2 точен. Отметим также, что для такого оператора $P(D)$ в работе [11] показано, что всякий компакт положительной меры Лебега, лежащий в $G$, неустраним

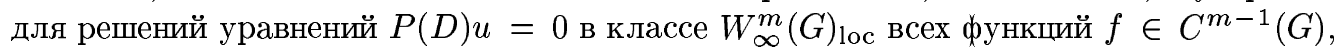
частные производные порядка $m-1$ которьх локально удовлетворяют в $G$ условию Липщица-Гёльдера с показателем 1 . Поскольку при любом $p>1$ справедливо включение $W_{\infty}^{m}(G)_{\text {lос }} \subset W_{p}^{m}(G)_{\text {loc }}$, отсюда следует, что при всех $p \in(1, \infty)$ и $s=m$ теоремы 1 и 2 перестают быть верньми.

В доказательстве теоремы 2 будет использовано одно вспомогательное утверждение. Для его формулировки напомним, что двоичным кубом в $\mathbb{R}^{n}$ назьвается всякий куб вида $\left[s_{1} 2^{-l},\left(s_{1}+1\right) 2^{-l}\right] \times \cdots \times\left[s_{n} 2^{-l},\left(s_{n}+1\right) 2^{-l}\right]$, где $s_{1}, \ldots, s_{n}$ - целые числа, $l$ натуральное. 
ЛЕмма 1 (Р. Харви и Дж. Полкинг [1], см. также [2, с. 20-22]). Пусть $\left\{Q\left(x_{j}, r_{j}\right)\right\}_{j=1}^{d}$, $d \in \mathbb{N},-$ конечное множество двоичных кубов, внутренности которых попарно не пересекаются. Тогда существует такое множество функиий $\left\{\phi_{j}\right\}_{j=1}^{d}$, бесконечное число раз дифференцируемых в $\mathbb{R}^{n}$ и принимающих только вещественные значения в интервале $[0,1]$, что при всех $j=1, \ldots, d$ носитель $\phi_{j}$ содержится в кубе $Q\left(x_{j}, \frac{3}{2} r_{j}\right)$, для любого мультииндекса $k \in \mathbb{N}_{0}$ справедлива очен$\kappa a\left|D^{k} \phi_{j}\right| \leqslant C(n,|k|) r_{j}^{-|k|}$, функция $\sum_{j=1}^{d} \phi_{j}(x)$ принимает значения в интервале $[0,1]$, причем на множестве $\bigcup_{j=1}^{d} Q\left(x_{j}, r_{j}\right)$ выполняется равенство

$$
\sum_{j=1}^{d} \phi_{j}(x)=1 .
$$

2. Доказательство теоремы 2. Пусть вьполнены условия теоремы 2 и пусть $f \in$

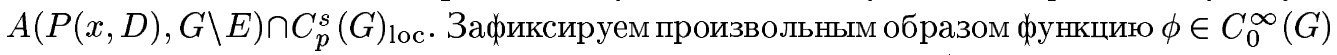
и действительное число $\varepsilon \in(0,1)$ и определим множество $E^{\phi}$ как пересечение $E$ с носителем функции $\phi$. Поскольку $E^{\phi}$ - компакт, существует такое покрытие этого множества конечным числом открытых шаров, что радиус каждого шара $<\varepsilon$, и величина, равная сумме этих радиусов, возведенных в степень $n-q(m-s)$, не превосходит $\operatorname{mes}^{n-q(m-s)} E+1$; а так как каждьй шар содержится в объединении не более чем $5^{n}$ двоичных кубов, длина ребра которых не превосходит радиуса этого шара, можно считать, что $E^{\phi}$ содержится в объединении конечного множества двоичных кубов $\left\{Q\left(x_{j}, r_{j}\right)\right\}_{j=1}^{d}, d \in \mathbb{N}$, с попарно не пересекаюшимися внутренностями и

$$
\sum_{j=1}^{d} r_{j}^{n-q(m-s)} \leqslant C(n, m, p, s, E)
$$

при этом, очевидно, можно предполагать, что при всех $j=1, \ldots, d$ куб $Q\left(x_{j}, 3 r_{j}\right)$ содержится в $\varepsilon$-окрестности $E_{\varepsilon}^{\phi}$ компакта $E^{\phi}$. Пусть $\left\{\phi_{j}\right\}_{j=1}^{d}$ - система функций из леммы 1 , образуюшая разбиение единицы для системы кубов $\left\{Q\left(x_{j}, r_{j}\right)\right\}_{j=1}^{d}$. Тогда, пользуясь тем, что $f \in A(P(x, D), G \backslash E)$ и носитель функции $\left(1-\sum_{j=1}^{d} \phi \phi_{j}\right)$ содержится в $G \backslash E$, имеем

$$
\begin{aligned}
|(P(x, D) f, \phi)| & =\left|\sum_{j=1}^{d}\left(P(x, D) f, \phi \phi_{j}\right)+\left(P(x, D) f, 1-\sum_{j=1}^{d} \phi \phi_{j}\right)\right| \\
& =\left|\sum_{j=1}^{d}\left(P(x, D) f, \phi \phi_{j}\right)\right| \leqslant \sum_{j=1}^{d}\left|\sum_{|k| \leqslant m}(-1)^{|k|} \int_{G} f D^{k}\left(a_{k} \phi \phi_{j}\right) d x\right| .
\end{aligned}
$$

Зафиксируем номер $j$ и мультииндекс $k,|k|>s$, и выберем в кубе $Q\left(x_{j}, r_{j}\right)$ точку $y_{j}$ так, чтобы

$$
\left(M_{s} f\left(y_{j}\right)\right)^{p} \leqslant 2 r_{j}^{-n} \int_{Q\left(x_{j}, r_{j}\right)}\left(M_{s} f(x)\right)^{p} d x .
$$


Тогда, пользуясь оценкой производной функции $\phi_{j}$ из леммы 1, получаем

$$
\begin{aligned}
\left|\int_{G} f D^{k}\left(a_{k} \phi \phi_{j}\right) d x\right| & =\left|\int_{Q\left(x_{j}, \frac{3}{2} r_{j}\right)} f D^{k}\left(a_{k} \phi \phi_{j}\right) d x\right| \\
& =\inf _{g \in \mathscr{P}_{[s]}}\left|\int_{Q\left(x_{j}, \frac{3}{2} r_{j}\right)}(f-g) D^{k}\left(a_{k} \phi \phi_{j}\right) d x\right| \\
& \leqslant C\left(\phi, a_{k}, n,|k|\right) r_{j}^{-|k|} \inf _{g \in \mathscr{P}_{[s]}}\left(\int_{Q\left(x_{j}, \frac{3}{2} r_{j}\right)}|f-g| d x\right) \\
& \leqslant C\left(\phi, a_{k}, n,|k|\right) r_{j}^{-|k|}\left|\inf _{g \in \mathscr{P}_{[s]}}\left(\int_{Q\left(y_{j}, 3 r_{j}\right)}|f-g| d x\right)\right| \\
& \leqslant C\left(\phi, a_{k}, n,|k|\right) r_{j}^{-|k|}\left(3 r_{j}\right)^{n} E\left(f, y_{j}, 3 r_{j}\right) \\
& \leqslant C\left(\phi, a_{k}, n,|k|\right) r_{j}^{-|k|}\left(3 r_{j}\right)^{n+s} M_{s} f\left(y_{j}\right) \\
& \leqslant C\left(\phi, a_{k}, n,|k|, s\right) r_{j}^{n+s-|k|}\left(r_{j}^{-n} \int_{Q\left(x_{j}, r_{j}\right)}\left(M_{s} f(x)\right)^{p} d x\right)^{1 / p} \\
& =C\left(\phi, a_{k}, n,|k|, s\right) r_{j}^{n / q+s-|k|}\left(\int_{Q\left(x_{j}, r_{j}\right)}\left(M_{s} f(x)\right)^{p} d x\right)^{1 / p}
\end{aligned}
$$

откуда, пользуясь неравенством Гёльдера, имеем

$$
\begin{aligned}
& \sum_{j=1}^{d}\left|\int_{G} f D^{k}\left(a_{k} \phi \phi_{j}\right) d x\right| \\
& \quad \leqslant \sum_{j=1}^{d} C\left(\phi, a_{k}, n,|k|, s\right) r_{j}^{n / q+s-|k|}\left(\int_{Q\left(x_{j}, r_{j}\right)}\left(M_{s} f(x)\right)^{p} d x\right)^{1 / p} \\
& \quad \leqslant C\left(\phi, a_{k}, n,|k|, s\right)\left(\sum_{j=1}^{d} r_{j}^{q(n / q+s-|k|)}\right)^{1 / q}\left(\sum_{j=1}^{d} \int_{Q\left(x_{j}, r_{j}\right)}\left(M_{s} f(x)\right)^{p} d x\right)^{1 / p} \\
& \quad \leqslant C\left(\phi, a_{k}, n,|k|, s\right)\left(\sum_{j=1}^{d} r_{j}^{n-q(|k|-s)}\right)^{1 / q}\left(\int_{E_{\varepsilon}^{\phi}}\left(M_{s} f(x)\right)^{p} d x\right)^{1 / p} \\
& \quad \leqslant C\left(\phi, a_{k}, n,|k|, s\right)\left(\sum_{j=1}^{d} r_{j}^{n-q(m-s)}\right)^{1 / q}\left(\int_{E_{\varepsilon}^{\phi}}\left(M_{s} f(x)\right)^{p} d x\right)^{1 / p} \\
& \quad \leqslant C(\phi, P(x, D), n, m, p, s, E)\left(\int_{E_{\varepsilon}^{\phi}}\left(M_{s} f(x)\right)^{p} d x\right)^{1 / p} \cdot
\end{aligned}
$$

Если $s$ натуральное и мультииндекс $k$ взят так, что $|k|=s<m$, то, используя включение $C_{p}^{s}(G)_{\text {loc }} \subset C_{p}^{s-1 / 2}(G)_{\text {loc }}$ (см. [3]) и производя вькладки, аналогичные приведенным 
выше, с заменой $s$ на $s-1 / 2$, получаем

$$
\begin{aligned}
& \sum_{j=1}^{d}\left|\int_{G} f D^{k}\left(a_{k} \phi \phi_{j}\right) d x\right| \\
& \quad \leqslant C\left(\phi, a_{k}, n, s\right)\left(\sum_{j=1}^{d} r_{j}^{n-q / 2}\right)^{1 / q}\left(\int_{E_{\varepsilon}^{\phi}}\left(M_{s-1 / 2} f(x)\right)^{p} d x\right)^{1 / p} \\
& \quad \leqslant C\left(\phi, a_{k}, n, s\right)\left(\sum_{j=1}^{d} r_{j}^{n-q(m-s)}\right)^{1 / q}\left(\int_{E_{\varepsilon}^{\phi}}\left(M_{s-1 / 2} f(x)\right)^{p} d x\right)^{1 / p} \\
& \quad \leqslant C(\phi, P(x, D), n, m, p, s, E)\left(\int_{E_{\varepsilon}^{\phi}}\left(M_{s-1 / 2} f(x)\right)^{p} d x\right)^{1 / p} .
\end{aligned}
$$

Пусть теперь мультииндекс $k$ удовлетворяет неравенству $|k|<s$. В этом случае функция $f$ имеет обобщенные производные по Соболеву всех порядков $\leqslant|k|$, локально интегрируемые в степени $p$ (см. [3]). Поэтому, используя лемму 1 , имеем

$$
\begin{aligned}
\sum_{j=1}^{d}\left|\int_{G} f D^{k}\left(a_{k} \phi \phi_{j}\right) d x\right| & =\sum_{j=1}^{d}\left|\int_{G}\left(D^{k} f\right) a_{k} \phi \phi_{j} d x\right| \leqslant \sum_{j=1}^{d} \int_{G}\left|D^{k} f\right| \cdot\left|a_{k} \phi\right| \cdot \phi_{j} d x \\
& \leqslant C(P(x, D), \phi) \sum_{j=1}^{d} \int_{G}\left|D^{k} f\right| \phi_{j} d x \\
& \leqslant C(P(x, D), \phi) \int_{G}\left|D^{k} f\right|\left(\sum_{j=1}^{d} \phi_{j}\right) d x \\
& \leqslant C(P(x, D), \phi) \int_{E_{\varepsilon}^{\phi}}\left|D^{k} f\right| d x
\end{aligned}
$$

Объединяя (4)-(7), получаем оценку

$$
\begin{aligned}
|(P(x, D) f, \phi)|= & \left|\sum_{j=1}^{d}\left(P(x, D) f, \phi \phi_{j}\right)\right| \leqslant \sum_{j=1}^{d}\left|\sum_{|k| \leqslant m}(-1)^{|k|} \int_{G} f D^{k}\left(a_{k} \phi \phi_{j}\right) d x\right| \\
\leqslant & C(\phi, P(x, D), n, m, p, s, E)\left(\left(\int_{E_{\varepsilon}^{\phi}}\left(M_{s} f\right)^{p} d x\right)^{1 / p}\right. \\
& \left.+\left(\int_{E_{\varepsilon}^{\phi}}\left(M_{s-1 / 2} f\right)^{p} d x\right)^{1 / p}+\sum_{|k|<s} \int_{E_{\varepsilon}^{\phi}}\left|D^{k} f\right| d x\right),
\end{aligned}
$$

где положено $M_{s} f \equiv 0$ в $G$ при $s \leqslant 0$. Поскольку $\operatorname{mes}^{n} E^{\phi}=0$, то $\operatorname{mes}^{n} E_{\varepsilon}^{\phi} \rightarrow 0$ при $\varepsilon \rightarrow 0$. Поэтому из абсолютной непрерьвности интеграла Лебега вытекает, что

$$
\left(\int_{E_{\varepsilon}^{\phi}}\left(M_{s} f\right)^{p} d x\right)^{1 / p}+\left(\int_{E_{\varepsilon}^{\phi}}\left(M_{s-1 / 2} f\right)^{p} d x\right)^{1 / p}+\sum_{|k|<s} \int_{E_{\varepsilon}^{\phi}}\left|D^{k} f\right| d x \rightarrow 0
$$


при $\varepsilon \rightarrow 0$. Следовательно, $(P(x, D) f, \phi)=0$. Поскольку в наших рассуждениях функция $\phi$ выбиралась произвольным образом, это означает, что $f \in A(P(x, D), G)$.

Теорема 2 доказана.

Заметим, что в приведенном доказательстве фактически использовалось лишь то, что пересечение множества $E$ с любым компактньм подмножеством области $G$ имеет конечную меру Хаусдорфа порядка $n-q(m-s)$.

\section{СПИСОК ЦИТИРОВАННОЙ ЛИТЕРАТУРЫ}

[1] Harvey R., Polking J. C. Removable singularities of solutions of linear partial differntial equations // Acta Math. 1970. V. 125. №1/2. P. 39-56.

[2] Тарханов Н.Н. Ряд Лорана для решений эллиптических систем. Новосибирск: Наука, 1991.

[3] Sharpley R., DeVore R. Maximal functions measuring smoothness // Mem. Amer. Math. Soc. 1984. V. 47. № 293. P. 1-113.

[4] Bojarski B. Sharp maximal operator of fractional order and Sobolev imbedding inequalities // Bull. Polish Acad. Sci. Math. 1985. V. 33. № 1-2. P. 7-16.

[5] Pokrovskii A. V. Removable singularities for solutions to elliptic equations in SharpleyDeVore classes // Функциональные пространства. Дифференциальные операторы. Проблемы математического образования. Тезисы докладов второй Международной конференции, посвященной 80-летию члена-корр. РАН профессора Л. Д. Кудрявцева. М.: Физматлит, 2003. С. 87.

[6] Zalcman L. Mean values and differential equations // Israel Math. J. 1973. V. 14. P. 339-352.

[7] Покровский А. В. Теоремы о среднем для решений линейных дифференциальных уравнений с частными производными // Матем. заметки. 1998. Т. 64. № 2. С. 260-272.

[8] Бесов О. В., Ильин В. П., Никольский С. М. Интегральные представления функций и теоремы вложения. М.: Наука, 1996.

[9] Kalderón A. P. Estimates for singular integral operators in terms of maximal functions // Studia Math. 1972. V. 44. P. 167-186.

[10] Покровский А. В. Об устранимых особенностях решений однородных эллиптических уравнений в классах Никольского-Бесова // Докл. РАН. 2001. Т. 380. № 2. С. 168-171.

[11] Verdera J. $C^{m}$-approximations by solutions of elliptic equations and Calderon-Zygmund operators // Duke Math. J. 1987. V. 55. № 1. P. 157-187.

Институт математики НАН Украины, г. Киев

Поступило

E-mail: pokrovsk@imath.kiev.ua

20.06 .2003

Исправленный вариант

13.09.2004 